In Cres. Vol. $3 N^{\circ} 1:$ pp. 23-29, 2012

\title{
LA APLICACIÓN DE LOS JUEGOS DIDÁCTICOS BASADOS EN EL ENFOQUE SIGNIFICATIVO MEJORA EL LOGRO DE APRENDIZAJE EN EL ÁREA DE MATEMÁTICA*
}

\author{
THE APPLICATION OF DIDACTIC GAMES BASED ON THE \\ MEANINGFUL APPROACH IMPROVES THE LEARNING \\ ACHIEVEMENT IN THE AREA OF MATHEMATICS
}

Jenny Maribel Lezama Ruiz ${ }^{1}$, Carla Cristina Tamayo Ly ${ }^{2}$

\section{RESUMEN}

La investigación tuvo como objetivo general determinar si la aplicación de los juegos didácticos basados en el enfoque significativo utilizando material concreto mejora el logro de aprendizaje en el área de Matemática de los estudiantes del tercer grado, sección única, de Educación Primaria, de la Institución Educativa "República Federal Socialista Yugoslavia”, de Nuevo Chimbote, en el año 2011.

Este estudio consistió en una investigación preexperimental. Se realizó con 12 estudiantes de la mencionada Institución Educativa en el año 2011. En el procesamiento de datos, se utilizó la estadística descriptiva para la interpretación de las variables, de acuerdo a los objetivos de la investigación. Asimismo, se utilizó la estadística no paramétrica en la prueba de Wilcoxon para comparar la mediana de dos muestras relacionadas.

Para la prueba de la hipótesis se utilizó el estadístico de contraste en la prueba de Wilcoxon, en la cual se pudo apreciar el valor de $\mathrm{P}=0,001<0,05$; es decir, existe una diferencia significativa en el nivel de logro de aprendizaje en el área de matemáticas obtenidos en el pretest y postest. Por lo tanto, se concluyó que la aplicación de los juegos didácticos basados en el enfoque significativo utilizando material concreto mejora significativamente el logro de aprendizaje en el área de Matemática de los estudiantes del tercer grado, sección única, de

* Recibido: 09 de enero del 2012; aceptado: 14 de junio del 2012.

1 Bachiller. Escuela Profesional de Educación, en la Carrera Profesional de Educación Primaria de la Universidad Católica Los Ángeles de Chimbote.

2 Licenciada en Educación Inicial, Docente Investigadora en la Escuela Profesional de Educación. Universidad Católica Los Ángeles de Chimbote. 
Educación Primaria, de la Institución Educativa "República Federal Socialista de Yugoslavia”, de Nuevo Chimbote, en el año 2011.

PalabRas Clave: Juegos didácticos, Enfoque significativo, Material concreto, Logro de aprendizaje, Área de Matemática.

\section{ABSTRACT}

The research aimed to determine whether the application of didactic game using concrete materials based on the meaningful approach, improves third year primary school students's learning achievement in the mathematics area of the República Federal Socialista de Yugoslavia School in Nuevo Chimbote 2011.

In this pre-experimental research participated 12 students. The data collection used the descriptive statistics to interpret the variables according to the research's objectives. The non parametric statistics considered the Wilcoxon Test to estimate the significant differences two samples' means related.

The test statistic was used to test the hypothesis with the Wilcoxon test where the value of $\mathrm{P}$ was apreciated $(\mathrm{P}=0,001<0,05)$. It indicates that there is a significant difference in the level of learning achievement in the mathematics area taken from the pre test and post test. The study concluded that the application of the didactic games using concrete materials based on the meaningful approach improves third year primary school students's learning achievement in the mathematics area of the República Federal Socialista de Yugoslavia School in Nuevo Chimbote 2011.

KEY WORDS: Didactic games, Meaningful approach, Concret material, Learning achievement, Mathematics area.

\section{INTRODUCCIÓN}

La matemática es, ante todo, una actividad mental, que exige la utilización de competencias cognitivas complejas por parte del estudiante y que necesitan ser desarrolladas en forma eficiente y eficaz por parte de los docentes de dicha disciplina. Es así, como el hacer y el pensar en matemática representa un verdadero desafío para los niños y niñas que recién comienzan a insertarse en la educación formal, para lo cual muchas veces no están dispuestos ni preparados a enfrentar. La metodología de enseñanza utilizada juega un rol fundamental en el proceso de construcción de los conocimientos que potencien el pensamiento matemático y, más aún, que incentiven el interés de aprendizaje. Es en este último punto donde se encontró la principal misión de la presente investigación: generar disposición en los alumnos y alumnas para aprender una disciplina que por años ha arrojado bajas calificaciones dentro del currículo escolar y la que más disgustos genera en alumnos y alumnas, quienes, al no poseer esta disposición, encuentran más difícil el camino del aprendizaje. 
Cuando se trabaja con el área de Matemática casi siempre se hace de manera tradicional y autoritaria, limitando al niño a hacer muchas cosas que puede experimentar directamente. Esto le resulta difícil de aprender debido a que no responde a sus intereses. ${ }^{1}$

\section{PROBLEMA}

¿Cómo la aplicación de los juegos didácticos basados en el enfoque significativo utilizando material concreto mejora el aprendizaje en el área de Matemática de los estudiantes del tercer grado, sección única de Educación Primaria, de la Institución Educativa "República Federal Socialista de Yugoslavia", de Nuevo Chimbote, en el año 2011?

\section{MATERIALES Y MÉTODOS}

La presente investigación fue de tipo cuantitativo. Se utilizó el diseño preexperimental, el cual se basa en la medición de la variable respuesta antes y después de la exposición del sujeto a la variable independiente. De esta forma, el sujeto es considerado como su propio control. ${ }^{2}$ La población muestral estuvo conformada por 12 alumnos de tercer grado, sección única, de Educación Primaria, de la Institución Educativa "República Federal Socialista de Yugoslavia", de Nuevo Chimbote.

Para la recolección de datos se aplicó la técnica de la encuesta, utilizando como instrumento el cuestionario aplicado a los estudiantes. El cuestionario estuvo organizado por 16 ejercicios, los cuales se han calificado de acuerdo al grado de dificultad en su realización. Los ejercicios aplicados estaban acordes con las capacidades a desarrollar en los alumnos y de acuerdo con las competencias que presenta el área de Matemática.

Para la medición de la variable estrategias didácticas, se determinó desde un enfoque integrador que comprende tres dimensiones: modalidad de organización de la enseñanza, enfoque metodológico de aprendizaje y utilización del recurso como soporte de aprendizaje. Asimismo, la variable logro de aprendizaje se categorizó en tres tipos tomando en consideración los baremos propuestos por el Diseño Curricular Nacional, ${ }^{3}$ el cual estipula que A es igual a logro previsto, $\mathrm{B}$ en proceso y $\mathrm{C}$ en inicio.

El procesamiento y análisis de datos se realizó a través de la estadística descriptiva e inferencial para la interpretación de las variables de acuerdo a los objetivos de la investigación. Asimismo, se utilizó la estadística no paramétrica en la prueba de Wilcoxon para comparar la mediana de dos muestras relacionadas y la contrastación de la hipótesis. 


\section{RESULTADOS}

EVALUAR El LOGRO DE APRENDIZAJE EN EL ÁREA DE MATEMÁTICA A TRAVÉS DE UN PRE TEST

\section{Tabla 01}

NIVEL DE LOGRO DE APRENDIZAJE EN EL ÁREA DE MATEMÁTICA DE LOS ESTUDIANTES DEL TERCER GRADO, SECCIÓN ÚNICA, DE EDUCACIÓN PRIMARIA, DE LA I.E. "REPÚBLICA FEDERAL SOCIALISTA DE YUGOSLAVIA", SEGÚN LAS CALIFICACIONES OBTENIDAS EN EL PRETEST, NUEVO CHIMBOTE, MARZO DE 2011

\begin{tabular}{ccc}
\hline $\begin{array}{c}\text { Niveles de logro } \\
\text { de aprendizaje }\end{array}$ & $\mathbf{N}^{\mathbf{0}}$ de estudiantes $\mathbf{f}_{\mathrm{i}}$ & Porcentaje (\%) \\
\hline A & 0 & 0 \\
B & 1 & 8 \\
C & 11 & 92 \\
\hline TOTAL & 12 & 100 \\
\hline
\end{tabular}

Fuente: Cuestionario aplicado en marzo del 2011.

En la tabla 01 se observa que el $92 \%$ de los estudiantes tuvo un nivel de logro de aprendizaje en inicio, es decir C; mientras que el $0 \%$ de los estudiantes obtuvo un nivel de logro de aprendizaje A; es decir un logro previsto.

ESTIMAR EL LOGRO DE APRENDIZAJE EN EL ÁREA DE MATEMÁTICA A TRAVÉS DE UN POST TEST

Tabla 02

NIVEL DE LOGRO DE APRENDIZAJE EN EL ÁREA DE MATEMÁTICA DE LOS ESTUDIANTES DEL TERCER GRADO, SECCIÓN ÚNICA, DE EDUCACIÓN PRIMARIA, DE LA I.E. "REPÚBLICA FEDERAL SOCIALISTA DE YUGOSLAVIA”, SEGÚN LAS CALIFICACIONES OBTENIDAS EN EL POSTEST, NUEVO CHIMBOTE, MARZO DE 2011

\begin{tabular}{ccc}
\hline $\begin{array}{c}\text { Niveles de logro } \\
\text { de aprendizaje }\end{array}$ & $\mathbf{N}^{\mathbf{0}}$ de estudiantes $\mathbf{f}_{\mathbf{i}}$ & Porcentaje (\%) \\
\hline A & 12 & 100 \\
B & 0 & 0 \\
C & 0 & 0 \\
\hline TOTAL & 12 & 100 \\
\hline
\end{tabular}

Fuente: Cuestionario aplicado en abril del 2011. 
En la tabla 02 , se observa que el $100 \%$ de los estudiantes tuvo un nivel de logro de aprendizaje A; es decir, un logro previsto, mientras que el $0 \%$ de los estudiantes obtuvo un nivel de logro de aprendizaje C; es decir, un nivel de logro en inicio.

EN RELACIÓN A LA HIPÓTESIS DE LA INVESTIGACIÓN

Tabla 03

ESTADÍSTICOS DE CONTRASTE

\begin{tabular}{lc}
\hline & Post test - Pre test \\
\hline$Z$ & $-3,357^{\mathrm{a}}$ \\
Sig. asintót. (bilateral) &, 001 \\
\hline $\begin{array}{l}\text { a. Basado en los rangos negativos. } \\
\text { b. Prueba de los rangos con signo de Wilcoxon. }\end{array}$
\end{tabular}

En la tabla 03 se puede apreciar que, según estadístico de contraste, prueba de Wilcoxon, el valor de $\mathrm{P}=0,001<0,05$; es decir, existe una diferencia significativa en el nivel de logro de aprendizaje en el área de Matemática obtenidos en el Pretest y Postest.

Por lo tanto, se concluyó que la aplicación de los juegos didácticos basados en el enfoque significativo utilizando material concreto, mejora significativamente el logro de aprendizaje en el área de Matemática de los estudiantes del tercer grado sección única de Educación Primaria, de la Institución Educativa "República Federal Socialista de Yugoslavia”, de Nuevo Chimbote, en el año 2011.

\section{DISCUSIÓN}

Al aplicar el instrumento de investigación a manera de pretest, los resultados demostraron que el $92 \%$ de los estudiantes tiene un logro de aprendizaje en inicio (C) de acuerdo al Diseño Curricular Nacional. ${ }^{3}$ Por otro lado, los resultados obtenidos evidencian que el $0 \%$ de los estudiantes tiene un logro de aprendizaje previsto (A), lo cual manifiesta que no han desarrollado las capacidades propuestas en el currículo y que los docentes no desarrollan actividades significativas que faciliten el logro de las mismas, tomando como referente a Dewey, ${ }^{4}$ quien señala que el conocimiento es algo fundamental para conseguir un aprendizaje significativo, aunque es crítico respecto a considerarlo un fin en sí mismo. 
$\mathrm{Al}$ aplicar el instrumento de investigación el cuestionario a manera de post test, los resultados demostraron que el $100 \%$ de los estudiantes tienen un nivel de logro de aprendizaje A; es decir, un logro previsto, mostrando que los estudiantes lograron desarrollar las capacidades propuestas, mientras que el $0 \%$ de los estudiantes tiene como nivel de logro de aprendizaje C; es decir, en inicio. Es así que, al aplicar los juegos didácticos, mejoran los resultados obtenidos en el cuestionario final, corroborando lo planteado por Ausubel, ${ }^{5}$ quien señala que el juego es un instrumento para que el maestro logre aprendizajes significativos. En lo referido al enfoque de aprendizaje significativo utilizado, el cual es defendido por Ausubel, ${ }^{6}$ quien plantea que la esencia del aprendizaje significativo reside en que ideas expresadas simbólicamente son relacionadas de modo no arbitrario, sino sustancial con lo que el alumno ya sabe, señaladamente, algún aspecto esencial de su estructura de conocimiento. Cabe resaltar el logro de aprendizaje que se obtuvo al utilizar material concreto en las sesiones. Al respecto, afirman Poveda R. y Morales Y. ${ }^{7}$ que, cuando se enseña una disciplina como la matemática, el material concreto se convierte en una herramienta que permite al estudiante saber lo que está haciendo, puesto que tiene la posibilidad de ver, tocar y sentir. La idea de usar material concreto en el aula es "trasladar" algunos conceptos abstractos de la matemática a la manipulación, de tal modo que a partir de esta experiencia sensorial los conceptos sean construidos y relacionados con la realidad.

Se determinó que hay una diferencia significativa entre la aplicación de los juegos didácticos basados en el enfoque significativo utilizando material concreto y el logro de aprendizaje en el área de Matemática, lo cual se puede apreciar según el estadístico de contraste para dos muestras relacionadas la prueba no paramétrica de Wilcoxon que el valor de $\mathrm{P}=0,001<0,05$; es decir, el programa aplicado mejoró el aprendizaje en el área de Matemática de los estudiantes del tercer grado sección única de Educación Primaria, de la Institución Educativa "República Federal Socialista de Yugoslavia", siendo corroborado estos resultados por Cabrera M., quien realizó una investigación sobre el "Uso de los juegos como estrategia pedagógica para la enseñanza de las operaciones aritméticas básicas de Matemática de cuarto grado en tres escuelas de Barcelona”, en la cual concluye que utilizar los juegos didácticos como estrategias didácticas produce en los estudiantes una satisfacción. Por eso se recomienda a los docentes que planifiquen sesiones teniendo en cuenta el factor lúdico al momento de aplicar sus sesiones de aprendizaje y que no las dejen de lado por considerarlas una pérdida de tiempo. 


\section{CONCLUSIONES}

Se observa que al evaluar el logro de aprendizaje en el área de Matemática, la mayoría de los estudiantes presentan un nivel de logro de aprendizaje en inicio $(\mathrm{C})$, siendo esto un reflejo de que la metodología utilizada no se relaciona con la configuración del logro de aprendizaje de los alumnos, generando que no se sientan motivados para lograr el desarrollo de habilidades necesarias que les permitan la mejora sustancial de las capacidades propuestas para el área.

Luego, al estimar el logro de aprendizaje en el área de matemática a través de un postest, se observa que la totalidad de los estudiantes presentan un logro previsto (A) en sus aprendizajes de Matemática. Se infiere que la estrategia didáctica utilizada se relaciona con el logro de aprendizaje de los estudiantes y que posibilita que ellos desarrollen las habilidades propuestas para el desarrollo de capacidades matemáticas.

Se concluye que se acepta la hipótesis de investigación: la aplicación de los juegos didácticos basados en el enfoque significativo utilizando material concreto, mejora significativamente el logro de aprendizaje en el área de Matemática de los estudiantes del tercer grado, sección única, de Educación Primaria, de la Institución Educativa "República Federal Socialista de Yugoslavia", de Nuevo Chimbote, en el año 2011.

\section{REFERENCIAS BIBLIOGRÁFICAS}

1 ESPARZA M. Las dificultades en el aprendizaje de las matemáticas. México. Ciencia-UANL, 2010.

2 LÓPEZ A, PÉREZ C. Evaluación de programas en psicología aplicada. 2 ed. Madrid. DYKINSON, 2005 .

3 Ministerio DE EduCACión. Diseño Curricular Nacional de Educación Básica Regular. Perú. Ministerio de Educación, 2009.

4 KLENOWSKi B .Desarrollo de portafolios para el aprendizaje y la evaluación. 2 ed. Madrid. Narcea, 2005.

5 BRINNITZER M. Juegos y técnicas de recreación. 4 ed. Buenos Aires. Bonum, 2008.

6 Zapata O. Aprender jugando en la escuela primaria. 2 ed. México. Pax México, 1995.

7 POVEdA R, Morales Y. Tecnología-material concreto: Una excelente combinación para la enseñanza y aprendizaje de la Matemática. [Tesis para obtener el grado de bachiller de educación]. Costa Rica. Universidad Nacional de Costa Rica, 2000.

8 CABRERA M. "Uso de los juegos como estrategia pedagógica para la enseñanza de las operaciones aritméticas básicas de matemática de cuarto grado en tres escuelas del área Barcelona Naricual. Propuesta de un diseño instruccional". [Trabajo de Grado no publicado]. Venezuela. Universidad Central de Venezuela, 2005. 
